\title{
Sensing Hand Interactions with Everyday Objects by Profiling Wrist Topography
}

\author{
Julius Cosmo Romeo Rudolph \\ julius.rudolph@student.hpi.uni- \\ potsdam.de \\ Tactual Labs \\ Toronto, Ontario, Canada \\ Hasso Plattner Institute \\ Potsdam, Germany \\ Ricardo Jota \\ jotacosta@gmail.com \\ Tactual Labs \\ Toronto, Ontario, Canada
}

\author{
David Holman \\ david.holman@tactuallabs.com \\ Tactual Labs \\ Toronto, Ontario, Canada
}

\author{
Bruno De Araujo \\ bruno.dearaujo@tactuallabs.com \\ Tactual Labs \\ Toronto, Ontario, Canada
}

\author{
Daniel Wigdor \\ daniel@dgp.toronto.edu \\ University of Toronto DGP \\ Toronto, Ontario, Canada
}

\author{
Valkyrie Savage \\ vasa@di.ku.dk \\ Tactual Labs \\ Toronto, Ontario, Canada \\ University of Copenhagen \\ Copenhagen, Denmark
}
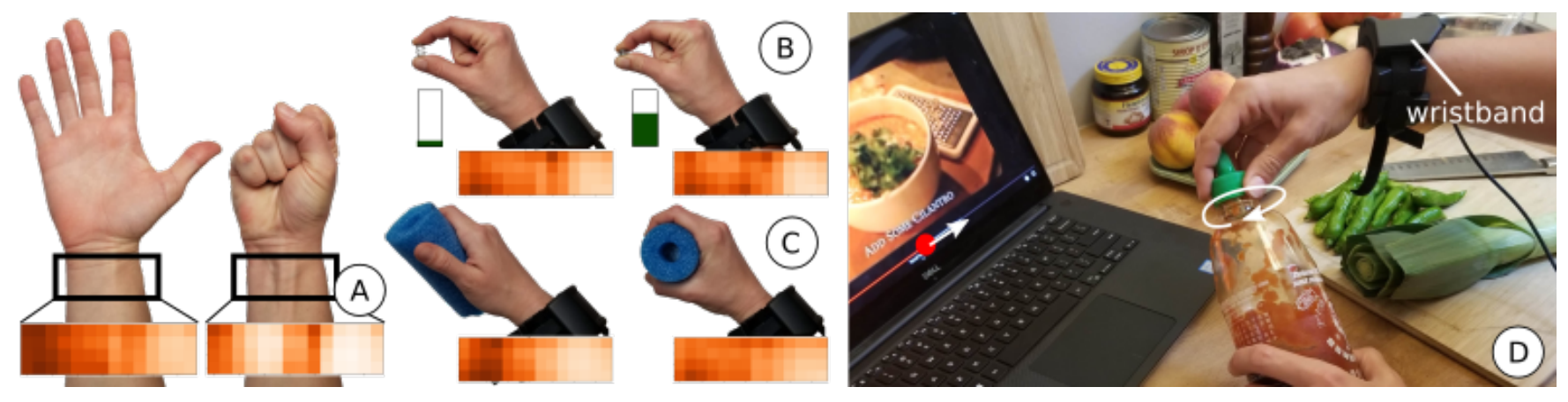

Figure 1: We use a capacitive sensing technique to measure changes in wrist profiles (A), which allows inferring the force (B) and grasp (C) used to interact with external objects. This enables digital input on unaugmented physical objects mediated by a wristband (D).

\begin{abstract}
We demonstrate rich inferences about unaugmented everyday objects and hand object interactions by measuring minute skin surface deformations at the wrist using a sensing technique based on capacitance. The wristband prototype infers muscle and tendon tension, pose, and motion, which we then map to force ( 9 users, $13.66+/-$ $9.84 \mathrm{~N}$ regression error on classes $0-49.1 \mathrm{~N}$ ), grasp (9 users, $81+/-7$ $\%$ classification accuracy on 6 grasps), and continuous interaction (10 users, $99+/-1 \%$ discrimination accuracy between 6 interactions, 89-97\% accuracy on 3 states within each interaction) using basic machine learning models.
\end{abstract}

Permission to make digital or hard copies of all or part of this work for personal or classroom use is granted without fee provided that copies are not made or distributed for profit or commercial advantage and that copies bear this notice and the full citation on the first page. Copyrights for components of this work owned by others than ACM must be honored. Abstracting with credit is permitted. To copy otherwise, or republish, to post on servers or to redistribute to lists, requires prior specific permission and/or a fee. Request permissions from permissions@acm.org.

TEI '22, February 13-16, 2022, Daejeon, Republic of Korea

(c) 2022 Association for Computing Machinery.

ACM ISBN 978-1-4503-9147-4/22/02 . \$15.00

https://doi.org/10.1145/3490149.3501320
We wrapped these sensing capabilities into a proof-of-concept end-to-end system, Ubiquitous Controls, that enables virtual range inputs by sensing continuous interactions with unaugmented objects. Eight users leveraged our system to control UI widgets (like sliders and dials) with object interactions (like "cutting with scissors" and "squeezing a ball"). Finally, we discuss the implications and opportunities of using hands as a ubiquitous sensor of our surroundings.

\section{CCS CONCEPTS}

- Human-centered computing $\rightarrow$ Interaction techniques; Ubiquitous and mobile computing systems and tools; Mixed / augmented reality; Interaction devices.

\section{KEYWORDS}

wrist topography, capacitive sensing, wristband, everyday objects, affordances

\section{ACM Reference Format:}

Julius Cosmo Romeo Rudolph, David Holman, Bruno De Araujo, Ricardo Jota, Daniel Wigdor, and Valkyrie Savage. 2022. Sensing Hand Interactions 
with Everyday Objects by Profiling Wrist Topography. In Sixteenth International Conference on Tangible, Embedded, and Embodied Interaction (TEI '22), February 13-16, 2022, Daejeon, Republic of Korea. ACM, New York, NY, USA, 14 pages. https://doi.org/10.1145/3490149.3501320

\section{INTRODUCTION}

As a wearable device on the wrist, smartwatches offer a useful vantage point to sense the prehensile movements of the hand. Recent research has demonstrated how sensing the fine-grained hand activity provides context about user activity and intent, and even object recognition. Prior work has demonstrated classifying repeated, fine hand motions via acceleration signals [40], as well as identifying objects held in the hand via EMG signals [17]. In this work, we examine how we can further increase contextual awareness about user activities and their environment: we infer properties of unaugmented everyday objects and the user's interaction with those objects by measuring continuous, instantaneous changes of wrist topography with capacitive signals.

Our wristband prototype infuses an electrical signal into the body and houses a capacitive sensor matrix to measure topographical changes at the wrist (see Figure 1). As the distance between a localized patch of skin and a sensor's receiver antenna varies, so does the capacitive charge. By measuring these minute skin surface deformations at multiple points near the wrist, it is possible to infer isometric muscle tension, tendon movement, and hand pose and motion.

Using machine learning, we map anatomical features like tendon tension, pose, and movement beneath the skin's surface to interaction properties like force, grasp, and object shape. Additionally, our approach enables discrimination of hand gestures and extends previous gesture recognition research by also precisely regressing the state within range interactions. Combining those sensing capabilities, this also enables ad hoc ubiquitous input controls leveraging the affordances of unaugmented objects: instead of instrumenting every water bottle and elastic band with touch or deformation sensors, we shift sensing to the wrist and examine the interaction between a hand and object. Sensing a hand's grasp and movement relative to an object means that users can appropriate an object's mechanical affordances as input for computational purposes-such as stretching a rubber band to zoom a map or twisting a bottle cap to adjust volume level.

Given our wristband prototype, the contributions of this paper are: (1) a sensing technique that measures wrist topography; (2) an evaluation of how topographical wrist profiles can enable inferring hand-object interactions; (3) an exemplar end-to-end system enabling ad-hoc virtual range inputs by sensing continuous hand interactions with everyday objects. The paper is structured as follows: we discuss related work, then present the hardware prototype including an evaluation of similarity between different users' wrist anatomy. Next, we describe a design space which links anatomical features at the wrist to derivable object and environmental properties and interactions; we evaluate inferring force from tension, grasp and object shape from pose, and dynamic interaction from motion through user studies. Through an end-to-end system and usability study, we demonstrate the technology's ability to distinguish gestures and to regress the state within interaction ranges for virtual input in ubiquitous environments.

\section{RELATED WORK}

Our work sits at the intersection of wrist-worn sensing and interaction with objects in ubiquitous environments.

\subsection{Wrist-Worn Sensing Techniques}

The wrist is an appealing location for sensing hands, as it does not interfere with dextrous finger use and does not suffer from external object occlusions. Prior work used smartwatches to monitor user activity and recognize objects in the user's environment, e.g., with electromagnetic signals [42], EMG [17], bio-acoustic signals [41], or audio plus inertial data [40]. This monitoring allows a portable system to display contextual information, but offers limited dynamic control of interactive systems. Others explore different sensing techniques to detect freehand gestures: Digits [37] leverages an infrared camera, Wristflex [15] uses an array of force sensitive resistors, Tomo [83] relies on electrical impedance tomography, Serendipity [76] and TapID [54] employ IMUs, SensIR [52] uses near-infrared sensing, and Capband [70] explores flexible capacitive sensors. Others use EMG [55], but given muscle locations this is more suited to the forearm [35]. Interferi [33] measures ultrasonic signals with piezoelectric transducers, and beyond recognizing gestures investigates continuous tracking tasks like regressing weights and smile intensity. Our work goes in a similar direction by focusing on properties of the interaction between hand and object, and additionally uses more-granular activity monitoring to precisely map pose and interaction to virtual controls. Another wearable system focused on properties of hand-object interaction is fSense[6], which uses photoplethysmography to discriminate two levels of force during common gestures.

While we also target a wristband form factor, we leverage wrist topography (broadly related to mechanomyography or MMG, first described by Grimaldi in 1665 [24]) to measure motions and hand activity. With MMG, an accelerometer, microphone, or high-accuracy laser near a muscle can "hear" its motion, and even infer fatigue [69]. MMG has been deeply explored in medical literature (for a review see [38] or [34]), but has seen little adoption in HCI, in spite of the fact that it has been shown to have a higher signalto-noise ratio than electromyography (EMG) [22] and improved sensing in locations away from the muscle belly [4]. Earlier approaches measured skin deformation [15, 20,27, 60, 70], but our band is tuned for fast-changing and highly-localized sub-millimeter wrist changes associated with grasping and interacting with objects, rather than global freehand gesture classification or on-body tapping. GestureWrist [60] presented 6 capacitive electrodes around the wrist to detect simple static poses; we go further with more receivers and more flexible detection. Photo-resistors have been also used to capture wrist contours, but are hard to scale; Fukui et al. [20] and ThumbSlide [2] used 75 and 16 photo resistors respectively and focused exclusively on capturing the index finger sliding along the thumb. EMPress [53] combined 4 force sensitive resistive (FSR) sensors with 4 EMG electrodes, and Liang et al. [44] used 5 capacitive pressure sensors. Liu, et. al. [47] explored accelerometerbased MMG to classify discrete gestures with a fixed wrist. While pressure- and inertial-based approaches use fewer sensors, they are still limited to a small set of gestures inadequate for capturing complex interactions with objects. Again, our work extends these 
earlier mechanomyography explorations by sensing force through tension, examining continuous range inputs in a ubiquitous environment, and using capacitance-based skin surface sensing easily scaled to high-detail wrist captures.

Some approaches have explored skin deformation and MMG principles at the back of the hand. Lin et al. [45] used four strain gauge sensors directly on top of the skin to detect hand gestures. Similarly, photo reflective sensor arrays on the back of the hand can measure detailed skin deformation for hand pose reconstruction [39, 68]; however these solutions impact hand mobility. More recently, vision-based systems with micro RGB $[78,81]$ or thermal cameras [31], mounted on the wrist facing the back of the hand, have been paired with $\mathrm{CNN}$-based models for hand reconstruction. While these enable freehand interactions, leverage wide lenses embedded in a watch [78], and can be tuned for complex static poses such as sign language [10], they are still sensitive to occlusion from object interactions or finger-crossing and cannot measure internal state such as muscle tension.

\subsection{Interaction with Objects in Ubiquitous Environments}

Ubiquitous computing [75] proposes a rich set of interaction paradigms that move away from earlier "natural" computing ideas [3] in which wall-based computers reacted to pointing and voice commands $[59,72]$. This newer vision suggests leveraging the affordances [21] of physical devices in UI design [65] and looking at everyday objects opportunistically as input devices [28]. Similar to Instant Controls (ICon) [12], Instant User Interfaces [13], and Ephemeral Interactions [74], we appropriate a wide range of everyday objects for interaction in our exemplar end-to-end system, but we avoid the need to physically augment the objects or environment. Object augmentation has been explored with visual markers $[12,28]$ and RFID tags [19]; alternatively, objects can be registered and tracked in real time using cameras [13, 74], but this limits the set of objects to those registered with the system and requires an instrumented environment.

Mobile devices can overcome this limitation, with their multiple portable sensors enabling interactive everyday objects to be used peripherally, for example in an office desk or kitchen tabletop environment [57]. Instrumenting the fingertip with a micro-camera [79] or an RFID reader [71] has also been explored. Smartwatches can enable rich multi-modal inputs using microphones-such as slapping and banging the forearm, blowing, or tapping the foot [29]-in situations where one or both hands are busy.

Movement in space around the body can enable proprioceptionenhanced inputs [11], further extending body-centric approaches [73] including for running or cycling [25]. Subtler interactions, like using the principles of magic to disguise embedded devices in everyday objects [1], are also possible, but again require instrumentation of the objects. While true micro-gesture- and grasp-based input has been examined through elicitation studies [5, 49, 64], it has been under-explored in implementation or achieved with limited setups like FingerInput's use of an overlooking depth camera [66]. Our approach removes those constraints by instrumenting the wrist instead of the object and focusing on the dynamic use of everyday objects. This is also related to Affordance++ [48]-but with a focus on objects as input rather than output from objects-and further extends the idea of hands as a controller [5].

Previous work has often used surface mapping to create UIs on surfaces $[26,30,77,84]$. We argue that this goes against the ubiquitous computing vision: these created overlays are similar to today's mobile devices, centralizing interaction on augmented surfaces. Annexing Reality [30] and Gripmarks [84] are closer to our presented end-to-end system. Annexing Reality re-targets proxy shapes in the environment for haptic feedback in VR, and Gripmarks focuses on passive-but-graspable objects. They both appropriate surfaces for interaction and focus on shape affordance, while our approach leverages objects' non-shape properties and interactive capabilities to enable ubiquitous inputs.

\section{SENSING SKIN SURFACE DEFORMATIONS AT THE WRIST}

\subsection{Capacitive Sensing at the Skin Surface}

Our proposed sensing technique uses parallel plate capacitors ${ }^{1}$ : capacitance $\mathbb{C} \propto \mathbb{A}$ surface area of the conductor plates and $\mathbb{C} \propto \frac{1}{d}$ the distance between the plates. We use the skin itself as a "plate": movement and deformation of the skin causes a change in $d$ (and thus $\mathbb{C}$ ). Unlike accelerometers used in $M M G[34,38]$, capacitors can measure changes in $d$ (e.g., vibration) without directly contacting the skin or dampening its motion. Laser-based range sensors can also measure displacement contact-free, but from a form factor perspective capacitive sensors are more compact than lasers.

\subsection{Sensing At the Wrist}

The wrist is our sensing location of choice: wrist-worn devices are unobtrusive and do not restrict hand motion or grasp. Additionally, watches are common wearable devices, which suggests that a sensing technique in this form factor has a path to wider adoption. With this location and form factor in mind, we discuss the input features that can be sensed at the wrist and relate them to underlying anatomical features.

The hand and fingers are controlled by tendons that pass along the anterior and posterior of the wrist to terminate with muscles in the forearm. The muscles and tendons of the upper limb typically exist in oppositional pairs, with flexors on the anterior of the hand, wrist, and forearm; and extensors on the posterior side. Abduction and adduction of the fingers is controlled by muscles intrinsic to the hand, i.e., that do not have a presence in the wrist. All thumb control tendons (flexion, extension, abduction, and adduction for all joints) pass through the wrist. The wrist proximal to the head of the ulna is home to the musculotendinous junction of these tendon/muscle pairs-the transition zone that connects pure muscle and pure tendon (see Figure 2).

When a muscle contracts and pulls a tendon, the musculotendinous junction moves, and the skin and subcutaneous fat layers nearby are pulled down into the space evacuated by the muscletendon unit. As this pliable potential space is filled or emptied, a user's skin surface topography changes. Localized uplift or subsistence of the epidermis corresponds to specific tendons. For example, the extensor pollicis brevis and -longus are responsible for thumb

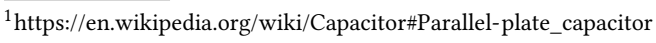




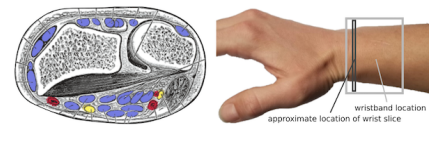

Figure 2: Wrist anatomy: nerves annotated in yellow, arteries in red, tendons in blue. Base image from Gray [23], public domain (left). Our wristband overlaps this anatomy (right).

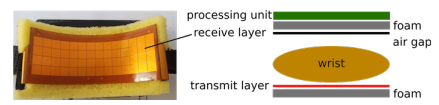

Figure 3: The heatmap receive layer (left) and our sensor stackup (right). A signal infused onto the skin from an anterior pad, along with the skin's uplift and subsidence, causes a change in $d$ relative to an array of electrodes above the wrist posterior.

extension; contractions of these muscles result in skin surface movement exhibited near the head of the radial bone. By referencing the underlying forearm anatomy, skin surface changes in this region can be mapped to thumb extension.

\subsection{Wristband Prototype}

Our wristband prototype, as noted, treats the skin as a plate in the parallel plate capacitor model. A high-frequency $(254 \mathrm{kHz})$ electrical signal is "infused" on the skin by a soft pad covered in conductive fabric on the anterior wrist, and received by a $4 \times 14$ matrix of printed, flexible electrodes (each $4.75 \mathrm{~mm}$ x $4.75 \mathrm{~mm}$, pitch $5 \mathrm{~mm}$ ) statically positioned $\approx 2 \mathrm{~mm}$ above the skin of the posterior wrist (see Figure 3 ). These reported dimensions are the design used in our final studies, namely the Interaction Study in Section 4.2.2 and the Application Study in Section 5. Our grasp and force studies in Sections 4.1.1 and 4.2.1 use a $3 \times 11$ matrix of $6 \mathrm{~mm}$ x $6 \mathrm{~mm}$ electrodes (less density), while the topographical profiles study reported in Section 3.5 uses a 15x4 matrix of $4.75 \mathrm{~mm}$ x $4.75 \mathrm{~mm}$ electrodes (wider overall). These changes represent our evolving understanding of device signals; we consider the theory of operation and the connection between skin-surface measurement and the environment as more important contributions than a particular wristband incarnation. The wristband prototype uses Tactual Labs' ${ }^{2}$ sensing platform, which provides high frame rate, low latency measurements of capacitive signal strength based on the work of Leigh, et al. [43].

Our studies focus mainly on extensor tendons, where the printed receivers are located. Flexor tendon motion is captured at a gross level: flexor motion changes wrist shape and coupling of the infusion pad, seen as a change in overall transmitted signal magnitude not localized to a particular matrix sensor. Inverting the prototype can swap the resolutions, but in conducting this work we observed the anterior skin has a larger range during interaction and more inter-user variation.

\subsection{Signals for Sensing}

Our prototype generates a 2-dimensional "heatmap" indicating physical location of 16-bit capacitive magnitude signals, sampled

\footnotetext{
${ }^{2}$ https://www.tactuallabs.com/
}
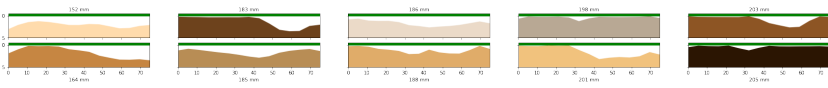

Figure 4: Topographical skin surface profiles for ten people, ordered from smallest to largest forearm circumference, across one row of the $75 \mathrm{~mm}$ sensor matrix. Profiles were recorded on right anterior wrist with fingers extended.
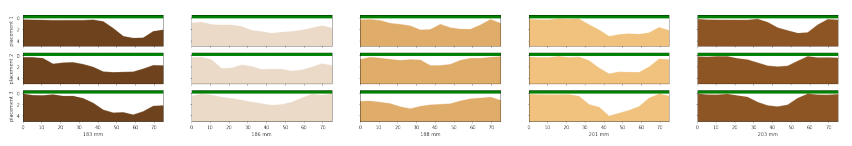

Figure 5: Topographical skin surface profiles for three placements (rows) on five participants (columns), across one row of the sensor. These were recorded on the right anterior wrist with fingers extended. Colors are for legibility and do not necessarily represent participant skin colours.

at $794 \mathrm{~Hz}$. The band streamed data to the PC via Tactual Labs' USB/Bluetooth drivers, which was processed using Python scripts.

Our data collection software, written in $\mathrm{C}++$, allows defining a gesture list and recording duration. When starting a recording, a user is presented with a description and/or video of the gesture to perform; clicking a button begins recording. During recording, a live but abstract visualization of captured heatmap data is displayed. The tool continuously receives band information, stores it in a buffer, and writes to a timestamped CSV when recording is done.

\subsection{Sensor Placement on the Wrist}

We conducted a pilot data collection to understand our sensor's sensitivity to different users and placements.

3.5.1 Procedure. Users placed the extra-wide $(15 \times 4)$ wristband with the matrix over the anterior of their right wrist. The wrist was held supinated (i.e., palm-up) with fingers extended flat. Users removed and replaced the band five times in the same location. We recorded one frame of data in each placement. Collections lasted less than 5 minutes.

We also used a conductive rod and gantry to test our sensor; measuring the rod's physical position relative to the sensor's reported signal allowed us to fit a power function mapping sensor signal magnitudes to distances from the sensor in $\mathrm{mm}$. We applied this to our recorded data for analysis and visualization.

3.5.2 Participants. From our organization we recruited 10 participants (2F, 8M). Wrist circumferences varied between 152 and 205 $\mathrm{mm}(\mathrm{Mdn}=186.5 \mathrm{~mm}, \mathrm{IQR}=16.75 \mathrm{~mm})$.

3.5.3 Results. We transformed our raw data into topographical maps with the aforementioned power function (see Figure 4). A pairwise Procrustes analysis ${ }^{3}$ between users' first placements yields disparities of $.01-.44(\mathrm{M}=.10, \mathrm{SD}=.09)$; this large disparity suggests that using skin-surface profiles necessitates per-user calibration.

\footnotetext{
${ }^{3}$ https://en.wikipedia.org/wiki/Procrustes_analysis ; this is often used in the biological sciences to measure similarity between organic structures of varying scale and translation
} 
Pair-wise Procrustes analysis within-users yields disparities of .00$.18(\mathrm{M}=.05, \mathrm{SD}=.04)$, suggesting similarity between placements but that models with small amounts of data may be unstable (see Figure 5). Between-user disparity was moderately correlated to wrist circumference difference, $\mathrm{r}(43)=.36, \mathrm{p}=.01$. We thus focus on single placements on single users as a base case for our technique. Also, our wide sensor, particularly on large wrists, seems to cause significant sensor-wrist contact at the ulnar and radial edges (while our goal is $2 \mathrm{~mm}$ hover): we narrow our sensor in later studies to focus on the tendons.

\section{INFERRING HAND INTERACTIONS FROM WRIST PROFILES}

We now turn our attention to changes in skin surface topography caused by mechanical displacement and vibration of muscle contractions, as well as uplift and subsidence of moving or tensing tendons. This is closely related to Mechanomyography (MMG). We discuss features of objects and the environment which can be inferred using skin surface topography: specifically, we look at how pose, motion, and tension can aid in sensing the shape of and user's interaction with an object. We thus explore a) measuring forces exerted at the fingertip, b) discriminating different grasps and gestures, and c) identifying states within ranges of gestures, across 3 user studies. All were conducted remotely in 2020/2021 during lockdown, leading to adaptations:

- users administered their own data collections using a mouse, and thus interactions were performed unimanually.

- each user had a personal, bespoke wristband, leading to signal and performance variation.

\subsection{Tension $\rightarrow$ Force}

Tendons and muscles are subjected to "loads" corresponding to weight or force, resulting in changed contraction profiles [67] or tension. Thus, musculotendinous activity is a proxy for force, which can be theoretically differentiated per finger (as finger tendons are independent). Load can be introduced on the muscles and tendons via either direct user actions (pressing on a table) or static object properties (the weight of a soup can).

4.1.1 Force Study. As our interest is a user's interaction with the world rather than the world's interaction with a user, we focus on user-generated forces.

Procedure: We used 9 small springs with different spring constants for which the length at maximum compression force was the same (.2"). Participants wore the band on the posterior of their dominant hand wrist, with their hand pronated and wrist held still. In each recording, forefinger and thumb were stably held .2" apart, and between them was either one of the 9 springs or nothing (i.e., 0 force required) (see Figure 6). Recording order was randomized, and we collected each condition 5 times for a total of 50 recordings. Each recording was $.5 \mathrm{~s}$ in length, and we collected 50 heatmap frames. Compression forces for the 9 springs ranged from 4.45-49.1 $\mathrm{N}(4.45 \mathrm{~N}, 8.4 \mathrm{~N}, 11.1 \mathrm{~N}, 22.3 \mathrm{~N}, 24.7 \mathrm{~N}, 30.3 \mathrm{~N}, 32.3 \mathrm{~N}, 44.7 \mathrm{~N}, 49.1$ $\mathrm{N})$, determined by what was available from McMaster-Carr at time of purchase. Based on user comments, we believe these adequately cover the comfortable range of forces for an average person's pinch.

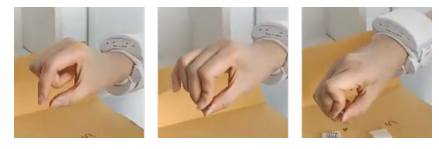

Figure 6: Users in our force study used 9 springs with different maximum compression forces, plus a "no spring" 0 force case, which were all held with index finger tip and thumb tip $.2 "(\approx 5 \mathrm{~mm})$ apart. Some users' hands visibly deformed under different forces, as can be seen here with one participant in the $0 \mathrm{~N}$ (left), $22 \mathrm{~N}$ (centre), and $49 \mathrm{~N}$ (right) cases.

Participants: From our organization, we recruited 9 users (2F, $7 \mathrm{M})$ whose ages range from $26-42(\mathrm{Mdn}=33, \mathrm{IQR}=4)$ and whose wrist sizes range from $143-198 \mathrm{~mm}(\mathrm{Mdn}=161.0 \mathrm{~mm}, \mathrm{SD}=15.44 \mathrm{~mm})$. All users were right-handed.

Results: Per-user compression force regression models had average error $13.66 \mathrm{~N}(\mathrm{SD}=9.84 \mathrm{~N})$ across all users.

Following Interferi's weight regression and extrapolation analysis [33], we first performed tests in which, for each user, one set of 10 recordings was left out and regressed from the other four sets. This gave average error $12.39 \mathrm{~N}(\mathrm{SD}=9.62 \mathrm{~N})$ using scikit-learn's MLPRegressor with default parameters, lbgfs solver, and tanh activation. We repeated the test, leaving out each force class and regressing it from the others using the same setup; this gave average error $13.66 \mathrm{~N}(\mathrm{SD}=9.84 \mathrm{~N})$. Although this error is relatively high, to our knowledge ours is the first method trying to derive pinch force with a hands-free wrist-based sensing approach, and we see value for discriminating more-tolerant force levels, e.g., button presses with different pressure intensities or rough assessment of strength. We expect these results to transfer to other grasps where users exert force involving other sets of fingers (e.g., squeezing a rubber ball or a sponge).

\subsection{Pose and Motion $\rightarrow$ Shape and Interaction}

Muscles and tendons in the wrist work together to move our fingers, and their pose correlates to hand pose, which is related to object shape. Their motion correlates to finger motion-whether userinitiated (I wiggle my finger) or environmentally-initiated (you wiggle my finger)-and to interaction patterns when an object or surface is involved.

We demonstrate mapping tendon and muscle pose and motion to user grasp, object shape, and interactions.

4.2.1 Grasp Study. While many grasp taxonomies exist [7, 14, 16, 46, 63], we focus on Feix, et al.,'s [18]. We selected grasps covering power and precision, as well as differing opposition structures. To ensure we were sensing grasp rather than weight, we fabricated all objects from identical weights of identical materials and only tested objects that could be held entirely within the hand (i.e., we did not use anchored objects). This limited the grasps that we were able to test, as the variance in objects' sizes to afford different grasps is large; we settled on 6 grasps using medium-sized objects.

Procedure: Using a uniform-density PlayDoh-like material, we created sets of four objects using molds and hand-shaping: a sphere (radius $4.25 \mathrm{~cm}$ ), a cylinder (length $15 \mathrm{~cm}$, radius $2.55 \mathrm{~cm}$ ), a plate (length $13 \mathrm{~cm}$, width $13 \mathrm{~cm}$, thickness $1.8 \mathrm{~cm}$ ), and a sphere with a 


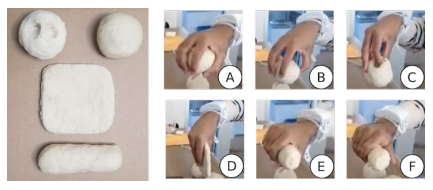

Figure 7: Users in our grasp study used 4 different molded objects (left) to perform 6 different grasps (right): power sphere (a), precision sphere (b), palmar pinch (c), parallel extension (d), medium wrap (e), and adducted thumb (f).

pinched lobe (radius $4.25 \mathrm{~cm}$ ). Each object comprised $300 \mathrm{~g}$ of material. We avoided 3D printing to ensure uniform material density. Each participant used a distinct set of objects for health reasons, but used the same set of 4 objects throughout their recording.

Participants wore the band on the posterior of their dominant hand wrist, with their hand pronated and wrist held still. Participants did sequential recordings: in each, they held one of the objects in a stable grasp: adducted thumb and medium wrap on the cylinder, power sphere and precision sphere on the sphere, parallel extension on the plate, and palmar pinch on the lobed sphere (see Figure 7). We recorded each grasp 10 times, for a total of 50 recordings. Each recording was $.5 \mathrm{~s}$, during which we recorded 50 heatmap frames.

Participants: From our organization, we recruited 9 users $(2 \mathrm{~F}$, $7 \mathrm{M})$ whose ages range from $26-42(\mathrm{Mdn}=33, \mathrm{IQR}=4)$ and whose wrist sizes range from $143-198 \mathrm{~mm}(\mathrm{Mdn}=161.0 \mathrm{~mm}, \mathrm{SD}=15.44 \mathrm{~mm})$. All users were right-handed.

Results: Per-user grasp classification had average accuracy 81 $\%(\mathrm{SD}=7 \%)$ across all users. Per-user held-object classification had a (predictably) higher average accuracy of $87 \%(\mathrm{SD}=7 \%)$ across all users (see Figure 8).

We used generic machine learning models from scikit-learn with default parameters, as our interest is in the data's presence rather than an optimally-designed model. We tested classification and regression trees (CART), linear discriminant analysis (LDA), logistic regression (LR), naïve Bayes (NB), linear support vector classification (LinearSVC), and support vector classification (SVC). For grasp classification, the best performers were $\mathrm{LR}(\mathrm{M}=79 \%, \mathrm{SD}=10$ $\%)$, LDA ( $M=80 \%, S D=11 \%)$, and LinearSVC $(M=81 \%, S D=9 \%)$. While LinearSVC was most accurate on average across all users, for some it was not the most accurate model; allowing the system to use the most accurate model for each user bumps average accuracy to $85 \%(\mathrm{SD}=9 \%)$ by mixing LinearSVC, SVC, LDA, and LR. For object classification, the results were, respectively, LinearSVC $(\mathrm{M}=84 \%, \mathrm{SD}=7 \%), \mathrm{LR}(\mathrm{M}=86 \%, \mathrm{SD}=6 \%)$, and $\mathrm{LDA}(\mathrm{M}=87 \%, \mathrm{SD}=7$ $\%$ ). Comparison to other wrist-based sensing techniques is difficult as they usually discriminate dynamic gestures (which we investigate in the following section) instead of static grasps on objects, or do not control for weight when discriminating held objects. Most comparable might be the work of Fan, et al., [17] with EMG: they achieve $85 \%$ mean accuracy across 6 grasps similar to ours, although without controlling object weight as we did. For object recognition their weight-controlled study discriminating 4 sizes of 3 object types (spheres, cylinders, and plates) had $35 \%$ recognition accuracy; this is arguably a harder classification task than ours since their different-sized objects are grasped in very similar ways.

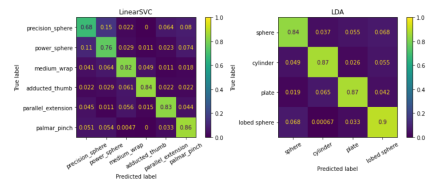

Figure 8: The confusion matrix for LinearSVC's grasp classification (left) showed generally satisfying to good results, with the most confusion coming from the power and precision grasps on the sphere. The confusion matrix for LDA's object classification (right) indicates the classifier had a reasonably strong ability to differentiate the objects.

Model tuning and the weight/density/material variation of realworld objects would likely further improve our results, but they indicate distinguishing static grasps on objects with reasonable accuracy is possible. We changed our sensor after this study from a $3 \times 11$ matrix to a $4 \times 14$ matrix; more, smaller pads improve locational specificity in the data to capture subtler interactions.

4.2.2 Interaction Study. The more interesting corollary of "can we distinguish static grasps on objects?" is, of course, "can we distinguish dynamic interactions with objects?" We examined how pose, motion, and force can combine to represent a user's interaction, and whether we could sense subtle changes in these dimensions from the wrist in a less-controlled scenario.

We focused on manipulative gestures ("whose intended purpose is to control some entity by applying a tight relationship between the actual movements of the gesturing hand/arm with the entity being manipulated" [58])-essentially the non-mediated version of "direct manipulation" [32] using real, physical objects. We looked explicitly at unimanual interactions for which the hand's pose and motion (not location in space) uniquely determine state: these types of interactions can be sensed with a single wristband.

Manipulative gestures are a sort of subcase of grasp, but we turned from Feix's grasp taxonomy to manipulation taxonomies, specifically Elliott and Connolly's "Dynamic Hand Movements" [16] and Bullock, et al.,'s "Within Hand Prehensile Manipulation Taxonomy" [7]. Both categorize single-handed prehensile manipulations (i.e., hand/object interaction requiring more than one finger). We merge them to examine the following (see Table 1):

- object contacts: moving (hand touchpoints slide or rotate relative to the manipulated object) or fixed (touchpoints on the manipulated object are stable) $[7,16]$

- opposition structure used: whether the force opposing the thumb is provided by the palm, finger pad(s), side of finger(s), or alternating contacts of side(s) and pad(s) [16]

- motion type: translation or rotation $[7,16]$

- axis of the opposition vector in the hand coordinate system: $\mathrm{X}, \mathrm{Y}$, or Z $[7,16]$

These dimensions can be extended from "describing the hand" to "using the hand as an input" by linking them with Card, et al.,'s classic input device taxonomy [8]. It organizes input device components by type and axis of movement (present in our manipulation taxonomy), and manipulation characteristics of the device; specifically whether using it causes positional or force change (a characteristic of the object being manipulated). Their final primitive is absolute 


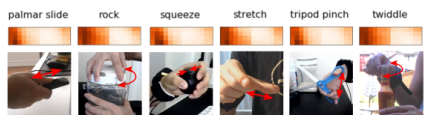

Figure 9: Object interactions with their heatmap signatures. Images from the usability study of Ubiquitous Controls.

or relative measurement: normally a physical characteristic of an input device, we propose that in extending object manipulation to interaction it is a software mapping problem, which we explore in our application (Section 5).

Procedure: Users gathered objects from around their home with slide, rock, twiddle, squeeze, stretch and tripod pinch affordances (see Figure 9). For each interaction we suggested various objects. Users wore their wristband on their dominant hand to perform prompted transitions using their objects. Collections lasted 45-90 minutes.

We tested multiple states for each interaction: for all but rock we collected training data on 3 states (min, middle, max). Due to rock's relatively larger range of motion, we collected 5 evenly-spaced states forming a superset of ( $\mathrm{min}$, middle, max). Users were invited to imagine a protractor or ruler aligned with their fingers to ensure state repeatability within a given interaction. Most objects were held entirely in the hand; for rock and twiddle the object (generally a jar or bottle) was set on the user's desk.

Recording: Users recorded 2-second transitions between each pair of states for each range interaction. For the 5 interactions with 3 states we recorded each transition 3 times $\left(\left(\begin{array}{l}3 \\ 2\end{array}\right)=6\right.$ transitions $x 5$ interactions $\mathrm{x} 3$ repetitions $=90$ recordings); for the 5 -state range, we recorded one full set of transitions $\left(\left(\begin{array}{l}5 \\ 2\end{array}\right)=20\right.$ recordings). This led to 110 total recordings per user. We used only the first and the last frame of the transitions as state samples for training and testing; this avoids overfitting consecutive frames, and we did not have continuous ground truth for intermediate states. For the 3-state ranges this yielded 36 samples ( 6 transitions $\times 3$ repetitions $\times 2$ frames), or 12 samples per state; for the 5 -state range we got 40 samples (20 transitions x 1 repetition x 2 frames), or 8 samples per state.

All transitions for an interaction were recorded together, but object order and transition order within the interaction were randomized. Across all interactions participants leveraged 10 unique objects' affordances, including reusing some objects for multiple affordances (see Table 1).

To assess if adding IMU information would improve classification results for dynamic interactions, we used an IMU 9DOF LSM9DS1 module with 3-axis accelerometer, 3-axis gyro, and 3-axis magnetometer in addition to the capacitive heatmap for this study. In contrast to the device-which samples at $794 \mathrm{~Hz}$-the IMU is sampled at $952 \mathrm{~Hz}$ and multiple frames are batched if available. The IMU outputs both raw 16-bit data signals and pre-processed Euler angles, and we configure our accelerometer for $\pm 2 g$ sensitivity for greatest distinctiveness in subtle motions.

Participants: We recruited 10 users $(2 \mathrm{~F}, 8 \mathrm{M})$ from within our organization, ranging in age from $25-41(\mathrm{Mdn}=32, \mathrm{IQR}=9.5)$, with wrist circumferences $143-198 \mathrm{~mm}(\mathrm{M}=165.5 \mathrm{~mm}, \mathrm{SD}=18.4 \mathrm{~mm})$.

Results: Using the same basic classifiers per-user described in the grasp study, we achieved $99 \%$ average accuracy across users discriminating interaction types and $90 \%$ classifying interaction state with LDA models. LDA achieves the best accuracies, which notionally makes sense as LDA assumes normal data distribution within and between classes-a natural fit with bodies and their motion. Training on IMU data as well improved interaction state classification accuracies only slightly $(+0.25 \%$ on average per user and interaction). For interaction type discrimination, average accuracies were $99 \%$ with or without IMU data. We hence report training results based solely on heatmap data in the following.

We evaluated the system's discrimination ability for two classification steps (classify interaction, classify state within interaction). We performed cross validation on each of the 6 interactions (6-fold, step 1) split by its number of states (step 2). Given the characteristics of our collection, this led to 8-fold cross-validation for rock and 12 -fold cross-validation for each of the remaining 5 interactions. Samples per state were evenly stratified, with 1 in each fold. Thus we tested 8 classification tasks: 1 to discriminate the 6 interaction types, and 7 to discriminate range states within individual interactions (6 3-state interactions + 15 -state rock).

We trained on just 10 samples per interaction type, selected in a stratified way across all states from the available sets of 36 or 40 samples. Our high mean accuracies of $99 \%(\mathrm{SD}=1 \%)$ within users are similar to accuracies of other techniques inferring dynamic gestures from biometric sensing at the wrist, although this comparison needs to be considered with care as others use different gesture sets and do not include object manipulation. For example, Tomo [83] achieves $97 \%$ accuracy on 10 hand gestures, Interferi [33] $93 \%$ on 11 gestures, and Maereg et al.'s infrared-based approach [51] $98 \%$ on 12 gestures, all within-users.

Models to identify state in the 53 -state interactions trained on 10/12 collected samples per state, while for the two versions of rock we had fewer recordings available: these within-interaction state classifiers trained on just $6 / 8$ collected samples per state. While 3-state gestures performed uniformly well across users with accuracies from $82-93 \%$, rock really excelled, with average classification accuracy $97 \%$ (see Table 2). Rock has a larger interaction range and includes wrist rotation-which registers strongly on our prototype due to broad motion of underlying anatomy-while in other interactions the wrist is more stable.

To assess if our technique performs differently for people with a higher BMI due to fat layers on the wrist, we correlated recognition accuracies and wrist size (a proxy for user BMI). Each of our studies had users with wrist circumference $>195 \mathrm{~mm}$ but we saw low correlation between size and accuracy ( $r^{2}$ values: .04 (force), .02 (grasp), .02 (shape), .02 (interaction)).

\section{UBIQUITOUS CONTROLS-LEVERAGING AFFORDANCES OF EVERYDAY OBJECTS TO CONTROL VIRTUAL RANGE INPUTS}

We see two major opportunities to apply understanding of object properties and interactions with objects: 1) implicit activity recognition to infer user behavior and everyday activities like "drinking water from a glass" and 2) explicit gesture input to precisely control a virtual system.

In this paper, we focus on the latter. We present Ubiquitous Controls, a unifying interactive system based on the studies in Section 4, 


\begin{tabular}{l|l|l|l|l|l|l} 
Interaction & Contacts & Opposition & Motion & Axis & Manipulation & Objects Used \\
\hline Palmar slide & Fixed & Palm & Translation & Y & Position & Box cutter, 2 playing cards \\
\hline Rock & Fixed & Pad & Rotation & Z & Position & Jar with lid \\
\hline Squeeze & Fixed & Palm & Translation & X & Force & $\begin{array}{l}\text { Plastic jar, plastic bottle, } \\
\text { stuffed animal, stress ball }\end{array}$ \\
\hline Stretch & Fixed & Finger & Translation & X & Force & Rubber band, hair tie \\
\hline Tripod pinch & Fixed & Fingers & Translation & X & Position & Pair of scissors \\
\hline Twiddle & Moving & $\begin{array}{l}\text { Alternating } \\
\text { Pad/Side }\end{array}$ & Rotation & Z & Position & Bottle, toothpaste tube
\end{tabular}

Table 1: Interaction descriptors for gestures explored in this work. Names from Elliott \& Connolly [16], except tripod pinch. All interactions are illustrated in Figure 9.

\begin{tabular}{l|c|c|c|c|c|c|c} 
Gesture & Rock & Rock (5) & Slide & Squeeze & Stretch & Tripod Pinch & Twiddle \\
\hline Accuracy & $97 \%$ & $82 \%$ & $90 \%$ & $89 \%$ & $89 \%$ & $93 \%$ & $89 \%$
\end{tabular}

Table 2: Each gesture tested in our interaction study and the classification accuracy achieved by LDA discriminating between its states, averaged across all 10 users. All gestures have 3 states except for rock (5).

which leverages the well-understood affordances of unaugmented everyday objects to provide guidance and haptic feedback for precise control of interactions. Many manipulative gestures are physically constrained to 1-dimensional motions within two extremes defined by an object's affordance (e.g., opening a pair of scissors, or sliding a box cutter), which makes them map well to virtual 1-dimensional inputs, like sliders or dials.

The system works by first differentiating between a variety of dynamic hand interactions with objects and then regressing the position within the range of the detected interaction. We developed a machine learning pipeline to run alongside the dedicated data collection software described in Section 3.4.

We now provide an interaction scenario, system overview, and evaluation with 8 participants controlling 3 widgets.

\subsection{Using Ubiquitous Controls}

A user reading a book on their couch wants to turn their music down. Instead of standing or searching for their phone, they perform a distinctive gesture to activate the Ubiquitous Controls system and twiddle the lid of their water bottle, at hand, counterclockwise to reach the desired volume (see Figure 10).

For the interaction to work, the user needs to configure their water bottle to be an input device; this task occurs only once per object, on-demand or ahead of time. They collect samples of their hand twiddling the cap to its extremes (i.e., far left, far right, centre): this data collection takes approximately 1 minute. The data are sent to a classification optimizer, which compares several machine learning configurations to select two models. One separates "twiddling a bottle cap" from other recorded controls, like "squeezing a toy" or "sliding a hair dryer selector." The second regresses the position of the bottle cap interaction based on the three recorded states.

After model training and selection, the system awaits the interactiontriggering gesture by analyzing live IMU information. Once detected, wrist profile and IMU data are sent to the first classifier to determine interaction, and to the interaction-specific model to regress range position. Finally, output is sent to the appropriate application: in this case, it adjusts the music player's volume level.

\subsection{Software Implementation}

Based on the results of our Interaction Study, for range position regression we collect 1-second recordings of holds in three positions: min, middle, and max.

Our training script collects the output csvs and extracts features. Cross-fold validation, per user, determines the best model. The live script takes this model and runs live data through it, outputting regression or classification information. Both scripts are written in Python with scikit-learn [56]. Our two model stages (1) classify high-level interactions and (2) regress a particular range location.

We use raw heatmap, accelerometer, and gyroscope values combined with 23 statistical features including $\mathrm{z}$-score, mean, and standard deviation. As in the study, we compare LDA, CART, LR, NB, and LinearSVC classifiers for gesture classification and use a linear regression model for range interpolation. We use standard configurations for all models. Trained models are n-fold cross-validated (where $\mathrm{n}=$ number of samples/number of trained states): the best classifier and the linear regression model are stored for live use.

In live mode, we detect a "double-flip" gesture, a placeholder activation trigger originally designed as a robust input-delimiter for mobile motion-based interactions [61], by thresholding the IMU gyro magnitude; the timeout for completing both flips is $300 \mathrm{~ms}$. Our system then constantly predicts interaction type and position until detecting the next "double-flip" gesture, which deactivates the interaction mode. The stored classifier determines the current interaction using single heatmap+IMU frames (see Figure 9 for example signatures).

We smooth regressed range position with an empirically-tuned 1-Euro Filter [9]. The live classification/regression script samples data from the band at $\approx 60 \mathrm{~Hz}$ and makes constant predictions in real time: we have achieved this on a variety of desktop and laptop computers, including a 2014 i7-4790 processor with $32 \mathrm{~GB}$ of memory. 


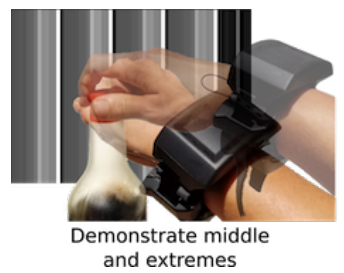
and extremes

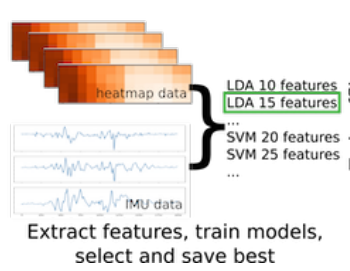

select and save best

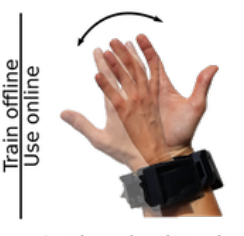

Await activation trigger

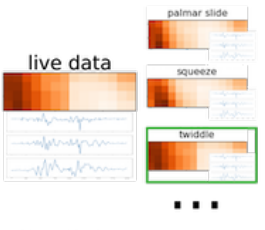

Classify interaction type

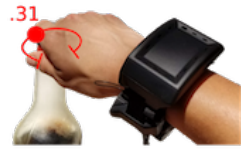

Regress range position

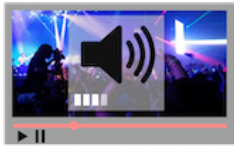

Use in application

Figure 10: The Ubiquitous Controls system: a user generates offline training data by recording the middle and extremes of an interaction. A script extracts features, trains several models, and selects and saves the best one. In online use, we look for a "double flip" activation gesture, then send data to saved models. We classify interaction type, then regress range position and smooth output. Finally, data is sent to an application.

Extracted data are sent to applications as input. We purposebuilt several widgets for Ubiquitous Controls, but with Sikuli [80] or other end-user programming techniques it would be possible to integrate such inputs into more applications.

\subsection{Evaluation}

We assessed the usability of our Ubiquitous Controls system in a real-time scenario with a study in which 8 users interacted with three widgets. Our primary goal was testing a live instance of our pipeline and whether it was functional to enable ubiquitous, continuous control input using affordances of everyday objects. Our hypothesis was that:

Hypothesis 1. The Ubiquitous Controls pipeline enables users to input interpolated range values within $1.5 \mathrm{~s}$

This is intended to mirror prior findings that users can take $1.172 \mathrm{~s}-1.233 \mathrm{~s}$ from stimulus to target acquisition using a trackpad with their dominant and non-dominant hand [36], or that a typist using an unfamiliar keyboard can require $1.2 \mathrm{~s}$ per key entry [50]. Participants completed input tasks in median $1.2 \mathrm{~s}$ per prompt, providing evidence for Hypothesis 1.

Asked about their preferred interaction technique, 3 users mentioned Ubiquitous Controls, 3 preferred freehand gestures, and 2 declared it depends upon the situation. Users reported state reproducibility and haptic feedback as favorable aspects of objectsupported interactions, suggesting our proposed interaction technique is a good alternative to freehand gestures for some kinds of input.

5.3.1 Procedure. We asked users to gather interactive objects from their homes; we provided them with a recommendation list illustrating different affordances. In contrast to the Interaction Study, we did not require specific affordances: users were explicitly allowed to use any objects they wanted as input controls. Studies lasted up to 60 minutes, exploring 3 tasks to be performed using both interaction methods (object-supported interactions and freehand gestures). Each participant wore a different wristband of the kind described in Section 3.3. A participant wore the same wristband for both input gesture techniques. The task consisted of controlling 3 widgets: a horizontal slider to "control the seek position of a video," a zoomable canvas to "control the zoom level of a map," and a knob to "control the volume of a stereo" (see Figure 11). Before each task, the experimenter described the widget and required users to define a freehand or object-supported gesture to control it, with users eventually doing both for all 3 widgets. The overall widget $\mathrm{x}$ interaction method configurations were randomized.

Every task consisted of five phases: a definition of the gesture's 3 distinct states ( $\max , \min$, and middle), a data collection, an instantaneous training, an experimentation phase where users could try out their trained model on the widget, and a test phase where users input prompted values as quickly as possible.

Based on the findings of the Interaction Study that 10 samples per state provided satisfying classification accuracies to discriminate 6 gestures and 3 states within each gesture, we had users collect 30 training samples per task (3 states $x 10$ repetitions). The collection comprised 1s-long static poses rather than transitions, and lasted roughly one minute for each 30 -sample set. The user then started a script to train a model with these samples, forcing them to put down their object or change their hand posture prior to the experimentation and test phases. The training script generated a linear regression model, which interpolated values within the range bounded by max and min; this permitted a richer, more continuous interaction compared to the 3-state classification models from the Interaction Study. The output of the regressor was not smoothed or post-processed, but was used raw to map inputs to the virtual control range. Prior to the recorded test phase, the user experimented with their trained model and its mapping to the $2 \mathrm{D}$ graphical widget for a maximum of two minutes. During this phase, if a user was not able to perform controlled manipulations (i.e., all inputs were regressed as "out of range," either above the trained maximum or below the trained minimum), we allowed them to recollect data and train again. The test phase consisted of a given number of virtual control value prompts to be matched within two minutes. Each prompt appeared the same number of times in a randomly-shuffled sequence. Holding the correct value for a fixed period of time triggered a transition between prompts. For the knob and zoomable canvas, users held 25 prompts for $.5 \mathrm{~s}$ each; with visual prompts and regressed model output both snapping to $1 / 5$ th of the overall input range. For the horizontal slider, users held 50 different prompts for $.5 \mathrm{~s}$ each, where each prompt was represented by an interval of $1 / 10$ th of the overall input range. Regressed values were presented continuously on the UI (see Figure 11). Performance metrics and sensed wristband data were captured during the study.

5.3.2 Participants. We recruited 8 users (1F, $7 \mathrm{M})$ within our institution. Their ages ranged from $24-45(\mathrm{Mdn}=33.5, \mathrm{IQR}=5.75)$ and their wrist circumferences from $152-210 \mathrm{~mm}(\mathrm{Mdn}=170.5 \mathrm{~mm}, \mathrm{SD}=19.4 \mathrm{~mm})$. 


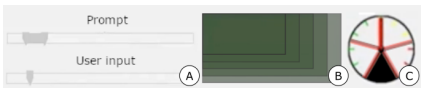

Figure 11: We used 3 UI widgets in the usability study to assess performance of our system: a horizontal slider (A), a zoomable canvas (B), and a rotary knob (C). For the slider the prompts were given in intervals and the output of the regressor was continuous (not snapped). The canvas and the knob featured visual "snapping" of both prompts and inputs to 5 evenly-spaced locations.

All but one of the studies was performed remotely, with the inperson study conducted in a physically-distanced manner outdoors.

5.3.3 Design. We recorded 23 trials for object-supported interactions ( 3 widgets $\times 7$ users +2 widgets $\times 1$ user) and 22 trials for freehand gestures ( 3 widgets $\mathrm{x} 6$ users +2 widgets $\mathrm{x} 2$ users). Due to timing and technical issues, 2 users could not complete the full study; one completed 2 widgets per interaction method and the other did not perform the final freehand interaction. In total, through 45 trials our users leveraged 13 unique object types, all 6 unique object interactions that we tested in our Interaction Study, and 8 unique freehand gestures for their controls. Of those 45 trials, the data collection and model training phases were repeated for 4 due to poor system performance during user experimentation. We define one complete test phase trial as 25 completed prompts within 120 seconds; this truncated the horizontal slider conditions from 50 prompts to balance our analysis.

\subsubsection{Results.}

Quantitative. With 407 matched prompts across all three widgets, most users could successfully control all widgets using objectsupported interactions. For 3 users input for the range slider with 10 buckets did not work during the study; without regression smoothing the input jitter made a $.5 \mathrm{~s}$ hold challenging. Users matched average 21.4 / 25 prompts $(M d n=25, S D=6.1)$ within the time limit, for interactions with at least one matched prompt. For such trials all but 2 per widget were finished in time.

We found strong support for Hypothesis 1: median input time with Ubiquitous Controls was $1.2 \mathrm{~s}$, with $58 \%$ of prompts completed within $1.5 \mathrm{~s}$ (not including the required $.5 \mathrm{~s}$ hold). As with other devices, more practice with the inputs and their behaviour would likely reduce input time. Though the slider had twice as many buckets as other widgets, no interaction was significantly slower or faster across trials with $\geq 1$ matched prompt.

Mean classification accuracy for the 3 range states was $92 \%$ $(\mathrm{Mdn}=97 \%, \mathrm{SD}=9 \%)$, similar to the Interaction Study. We also included IMU data alongside heatmap data for training and testing. Similar to the Interaction Study, removing IMU snapshots reduces performance only slightly $(-0.41 \%$ on average per user in post hoc analysis). Mean range interpolation error was $16 \%(\mathrm{Mdn}=10 \%$, $\mathrm{SD}=14 \%$ ), similar to Interferi [33]. This error also represents user interpretation error: users estimated state positions during data collection and we had no ground truth. For interaction discrimination, the mean accuracy of $84 \%$ ( $84 \%$ without IMU, Mdn=99\%, SD=28\%) was lower than in the Interaction Study; as some participants used

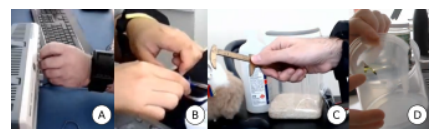

Figure 12: Users in our Ubiquitous Controls evaluation had various interesting interaction strategies. One used an unpowered oscilloscope (A). Another used both hands to precision-set a combination lock (B). Another precision input was a non-digital pair of brass calipers $(C)$. One user's "squeeze" object, a thin-walled plastic cup, broke through use as an input (D).

the same object-supported interactions for different interaction tasks, we believe the median of $99 \%$ is a more appropriate metric.

Users were also able to control the widgets using freehand gestures; there were no significant differences between objectsupported and freehand gestures in input time, number of matched prompts, model fit, or interaction type discrimination accuracy.

Qualitative. Participants' preferences about the interaction methods were mixed: three users preferred object-supported interactions, three preferred freehand gestures, and two declared it depends on the situation. Many users highlighted state reproducibility and haptic feedback, saying the "force made it easier to control" (P7) pinching with a rubber band versus freehand, or that feedback from a screw-top lid "lets you know where you are on the thing" (P4).

Many users explicitly mapped an object interaction or gesture to a widget's visual appearance or existing use in the wild. Users mentioned "if it's a video player, I'd like it to be linear" (P4), or "I've turned the knob of a stereo, so I know that a bottle cap is more consistent with the experience [than a linear control]" (P3). Our system's treatment of object-supported interactions did always not map well to users' understanding of how inputs work. For example, P1 used a knob on an unpowered oscilloscope as an input: in training, they twisted using mainly their wrist, and in testing with mainly their fingers. In normal use, these methods behave identically, but in our system they do not (see Figure 12A).

On using objects as inputs, one user suggested they would carry a "collection of favourite objects that were comfortable to use," or a multi-function input object (e.g., fidget cube) designed for this type of interaction (P6). Conversely, another mentioned that any object they have has to be "worthy of carrying with me," suggesting that an object only for input would not be worth the space in their bag or pockets (P2). In the end, it seems both object-supported and freehand gestures have a role in pervasive interaction, but what defines their roles requires further study.

\section{DISCUSSION AND LIMITATIONS}

We have demonstrated inference of object properties using capacitive topographical changes at the wrist, including an end-to-end system leveraging them for explicit ubiquitous input. As demonstrated through our studies on force, grasp, and interaction, tapping into our body's own actuation systems to detect isometric tension, pose, and movement re-imagines the hand as a sensor instead of integrating electronics into every object we wish to imbue with 
interaction. This also leads to the challenge of anatomical differences between users, which we showed extends beyond wrist circumference to wrist profiles. We now summarize challenges and opportunities that surfaced in executing this research.

\subsection{Performance}

While 3-state classification and 5-state interpolation with our prototype pipeline was usable, users' experiences with 10-state interpolation were mixed, and $4.9 \%$ of successful object-mediated inputs with Ubiquitous Controls took over 10 seconds to complete. We anecdotally noted these issues were often due to regression instability. In the study, we did not filter regressed data: smoothing with an empirically-tuned 1-Euro Filter [9] has dramatically improved system usability. A post hoc analysis on recorded frames from the study reduced mean "input" times by $.21 \mathrm{~s}$, even accounting for lag introduced by smoothing, and 1 percentage point fewer trials took $>10$ s to complete. The filter reduced regressor noise, but further work is required to ensure robust, continuous, and precise input.

Our sensor design evolved throughout the work and we used an IMU for some experiments. Post hoc analysis revealed IMU contribution to accuracy was minor ( $+.25 \%$ in the Interaction Study, $+.41 \%$ in the Application Study), and future work can explore new capacitive matrix geometries and densities to improve accuracy.

While our first study shows anatomical differences between people, we believe it possible to build a population-level machinelearning filter to separate arm/wrist motion and position from finger motion and position in order to better match users' mental models of how Ubiquitous Controls inputs work and to make interactions transfer across users. This would require significant data collections on people of varying wrist characteristics and is outside the scope of the present contribution.

\subsection{Midas Touch}

As computation moves to the body and everyday objects may serve both analog and digital functions, we encounter the so-called "Midas Touch" problem, where a system must distinguish between functional interaction and intentional input with an object [13]. In our prototype system, we use double-flip to demarcate these modes, but further exploration may find contextual or physiological (e.g., EEG) signals to distinguish whether a user twisting a bottle lid intends to take a drink or control their podcast. The ideal solution does not require a specific initiation gesture, but this is dependent upon the available cues. Multi-modal techniques like voice or eye-tracking might be required to disambiguate functional interaction and intentional input. Without extra modalities, a system could track events before and after recognition to disambiguate usage, however this introduces input delays as the system waits for differentiating events after recognizing an action.

\subsection{Beyond Shape and Force}

Better understanding the objects a user is interacting with is an obvious future direction that builds on the primitives of shape, force, and gesture. Force combined with pose and motion may reveal surface hardness, springiness, or mechanical stickiness. Touch on a held object from another hand may also be visible [82]. Taking motion's derivative, acceleration profiles during object interaction may correlate to weight, centre of mass, or change in weight distribution (e.g., if the object contains liquid).

\subsection{Reuse and Assemblage of Other Devices}

A corollary of re-purposing existing object properties is reprogramming existing input devices for new uses. After all, a disconnected game controller is just a high-quality fidget cube: both are primed with interesting physical mappings. A user could map the brightness of their room to the roll of a mouse wheel, adjust which cooktop burner is active by thumbing a joystick, or reuse a favourite PS/2 peripheral with a new machine without an adapter.

For accessibility reasons, allowing a user to reuse an object or input device they are familiar with for new and arbitrary interactions is a boon. Relocating a component's sensing from the device to the user also enables a new way to prototype input devices: assemblage of existing affordances held together by clay or tape is easy to reconfigure and immediately test, similar to the concept of Makers' Marks [62].

\subsection{Dynamic and Configurable Mapping}

The distributed nature of input and output also introduces challenges of mapping, for which we need new paradigms in end user programming that allow tying a specific input to a specific output.

All our tested interactions include explicitly-coded mappings, with the minimum value of a physical range permanently connected to the minimum value of a digital range, and all interactions are 1:1. More subtlety may be desirable: the abilities to map sub-ranges, to dynamically map physical and digital inputs, or to choose a different gesture according to the situation (working out vs. lying in bed) each contribute to a personal mapping experience where the interface bends to meet the user's intentions and is not bounded by virtual inputs or specialized input devices.

Meaningful, pervasive mapping techniques with Ubiquitous Controls also open opportunities for more hygienic and personal interactions with public displays or infrastructure: one's own items can be leveraged in lieu of a public touchscreen or other input device.

\section{CONCLUSION}

We have proposed a capacitance-based sensing method to extract the surface profiles of anatomical structures in a user's wrist; further we described our prototype implementation of a wristband using this sensing technique. We discussed the implications of the actual anatomy being sensed: that tendon and muscle tension, pose, and motion in the location of our wristband can be mapped to, respectively, force, grasp, and interaction of the hand and fingers with external objects. We demonstrated these mappings through several atomic studies. Finally, we describe Ubiquitous Controls, an end-to-end system that uses these capabilities to let users control virtual range inputs by interacting with unaugmented objects, and we conclude with a discussion of the implications and opportunities of using the hand as a sensor. We anticipate that using anatomy to understand interactions with and properties of everyday objects will provide a powerful framework for ubiquitous computing. 


\section{ACKNOWLEDGMENTS}

We would like to thank Maria Ruiz Maya, Goutham Palaniappan, and Kevin Stager for their assistance in preparing (and repairing) hardware prototypes; Myles Gifford, Pablo Saldarriaga, Michael Mohan, and Peter Hamilton for their support of the software tools, all our anonymous participants, and Cliff Forlines for his guidance and shepherding of the project.

\section{REFERENCES}

[1] Fraser Anderson, Tovi Grossman, Daniel Wigdor, and George Fitzmaurice. 2015 Supporting Subtlety with Deceptive Devices and Illusory Interactions. In Proceedings of the 33rd Annual ACM Conference on Human Factors in Computing Systems (Seoul, Republic of Korea) (CHI '15). Association for Computing Machinery, New York, NY, USA, 1489-1498. https://doi.org/10.1145/2702123.2702336

[2] Shuhei Aoyama, Buntarou Shizuki, and Jiro Tanaka. 2016. ThumbSlide: An Interaction Technique for Smartwatches Using a Thumb Slide Movement. In Proceedings of the $2016 \mathrm{CHI}$ Conference Extended Abstracts on Human Factors in Computing Systems (San Jose, California, USA) (CHI EA '16). Association for Computing Machinery, New York, NY, USA, 2403-2409. https://doi.org/10.1145/ 2851581.2892435

[3] Richard A. Bolt. 1980. "Put-That-There": Voice and Gesture at the Graphics Interface. SIGGRAPH Comput. Graph. 14, 3 (July 1980), 262-270. https://doi.org/ 10.1145/965105.807503

[4] Riley Booth. 2017. Design and Testing of a Wristband with Piezoelectric Sensors for Finger Gesture Recognition. Master's thesis. University of Calgary, Calgary, AB http://dx.doi.org/10.11575/PRISM/25577

[5] Idil Bostan, Oguz Turan Buruk, Mert Canat, Mustafa Ozan Tezcan, Celalettin Yurdakul, Tilbe Göksun, and Oğuzhan Özcan. 2017. Hands as a Controller: User Preferences for Hand Specific On-Skin Gestures. In Proceedings of the 2017 Conference on Designing Interactive Systems (Edinburgh, United Kingdom) (DIS '17). Association for Computing Machinery, New York, NY, USA, 1123-1134. https://doi.org/10.1145/3064663.3064766

[6] Thisum Buddhika, Haimo Zhang, Samantha Chan, Vipula Dissanayake, Suranga Nanayakkara, and Roger Zimmermann. 2019. fSense: Unlocking the Dimension of Force for Gestural Interactions using Smartwatch PPG Sensor. 1-5. https: //doi.org/10.1145/3311823.3311839

[7] Ian Bullock, Raymond Ma, and Aaron Dollar. 2013. A Hand-Centric Classification of Human and Robot Dexterous Manipulation. Haptics, IEEE Transactions on 6 (04 2013), 129-144. https://doi.org/10.1109/TOH.2012.53

[8] Stuart K. Card, Jock D. Mackinlay, and George G. Robertson. 1990. The Design Space of Input Devices. In Proceedings of the SIGCHI Conference on Human Factors in Computing Systems (Seattle, Washington, USA) (CHI '90). Association for Computing Machinery, New York, NY, USA, 117-124. https://doi.org/10.1145/ 97243.97263

[9] Géry Casiez, Nicolas Roussel, and Daniel Vogel. 2012. $1 €$ Filter: A Simple SpeedBased Low-Pass Filter for Noisy Input in Interactive Systems. In Proceedings of the SIGCHI Conference on Human Factors in Computing Systems (Austin, Texas, USA) (CHI '12). Association for Computing Machinery, New York, NY, USA, 2527-2530. https://doi.org/10.1145/2207676.2208639

[10] Feiyu Chen, Jia Deng, Zhibo Pang, Majid Baghaei Nejad, Huayong Yang, and Geng Yang. 2018. Finger Angle-Based Hand Gesture Recognition for Smart Infrastructure Using Wearable Wrist-Worn Camera. Applied Sciences 8, 3 (2018) https://doi.org/10.3390/app8030369

[11] Xiang "Anthony" Chen, Julia Schwarz, Chris Harrison, Jennifer Mankoff, and Scott Hudson. 2014. Around-Body Interaction: Sensing \& Interaction Techniques for Proprioception-Enhanced Input with Mobile Devices. In Proceedings of the 16th International Conference on Human-Computer Interaction with Mobile Devices \& Services (Toronto, ON, Canada) (MobileHCI '14). Association for Computing Machinery, New York, NY, USA, 287-290. https://doi.org/10.1145/2628363.2628402

[12] Kai-Yin Cheng, Rong-Hao Liang, Bing-Yu Chen, Rung-Huei Laing, and Sy-Yen Kuo. 2010. ICon: Utilizing Everyday Objects as Additional, Auxiliary and Instant Tabletop Controllers. In Proceedings of the SIGCHI Conference on Human Factors in Computing Systems (Atlanta, Georgia, USA) (CHI '10). Association for Computing Machinery, New York, NY, USA, 1155-1164. https://doi.org/10.1145/1753326. 1753499

[13] Christian Corsten, Ignacio Avellino, Max Möllers, and Jan Borchers. 2013. Instant User Interfaces: Repurposing Everyday Objects as Input Devices. In Proceedings of the 2013 ACM International Conference on Interactive Tabletops and Surfaces (St. Andrews, Scotland, United Kingdom) (ITS '13). Association for Computing Machinery, New York, NY, USA, 71-80. https://doi.org/10.1145/2512349.2512799

[14] M. R. Cutkosky. 1989. On grasp choice, grasp models, and the design of hands for manufacturing tasks. IEEE Transactions on Robotics and Automation 5, 3 (1989), 269-279.
[15] Artem Dementyev and Joseph A. Paradiso. 2014. WristFlex: Low-power gesture input with wrist-worn pressure sensors. UIST 2014 - Proceedings of the 27th Annual ACM Symposium on User Interface Software and Technology, 161-166. https://doi.org/10.1145/2642918.2647396

[16] J. M. Elliott and K. J. Connolly. 1984. A Classification of Manipulative Hand Movements. Developmental Medicine \& Child Neurology 26, 3 (1984), 283-296. https://doi.org/10.1111/j.1469-8749.1984. tb04445.x arXiv:https://onlinelibrary.wiley.com/doi/pdf/10.1111/j.14698749.1984.tb04445.x

[17] Junjun Fan, Xiangmin Fan, Feng Tian, Yang Li, Zitao Liu, Wei Sun, and Hongan Wang. 2018. What is That in Your Hand? Recognizing Grasped Objects via Forearm Electromyography Sensing. Proc. ACM Interact. Mob. Wearable Ubiquitous Technol. 2, 4, Article 161 (Dec. 2018), 24 pages. https://doi.org/10.1145/3287039

[18] Thomas Feix, Javier Romero, Heinz Bodo Schmiedmayer, Aaron M. Dollar, and Danica Kragic. 2016. The GRASP Taxonomy of Human Grasp Types. IEEE Transactions on Human-Machine Systems 46 (2 2016), 66-77. Issue 1. https: //doi.org/10.1109/THMS.2015.2470657

[19] Assaf Feldman, Emmanuel Munguia Tapia, Sajid Sadi, Pattie Maes, and Chris Schmandt. 2005. ReachMedia: On-the-move interaction with everyday objects. Proceedings - International Symposium on Wearable Computers, ISWC 2005, 52-59. https://doi.org/10.1109/ISWC.2005.44

[20] Rui Fukui, Masahiko Watanabe, Tomoaki Gyota, Masamichi Shimosaka, and Tomomasa Sato. 2011. Hand Shape Classification with a Wrist Contour Sensor: Development of a Prototype Device. In Proceedings of the 13th International Conference on Ubiquitous Computing (Beijing, China) (UbiComp '11). Association for Computing Machinery, New York, NY, USA, 311-314. https://doi.org/10. $1145 / 2030112.2030154$

[21] William W. Gaver. 1991. Technology Affordances. In Proceedings of the SIGCHI Conference on Human Factors in Computing Systems (New Orleans, Louisiana, USA) (CHI '91). Association for Computing Machinery, New York, NY, USA, 79-84. https://doi.org/10.1145/108844.108856

[22] Y. Geng, Liang Chen, Lan Tian, and G. Li. 2012. Comparison of electromyography and mechanomyogram in control of prosthetic system in multiple limb positions. In Proceedings of 2012 IEEE-EMBS International Conference on Biomedical and Health Informatics. 788-791.

[23] Henry Gray. 1918. Anatomy : descriptive and surgical (20 ed.). Lea and Febiger, New York.

[24] Franceso Maria Grimaldo. 1665. Physico-mathesis de lumine, coloribus, et iride, aliisque adnexis libri duo. Bologna, Italy.

[25] Nur Al-huda Hamdan, Ravi Kanth Kosuru, Christian Corsten, and Jan Borchers. 2017. Run\&;Tap: Investigation of On-Body Tapping for Runners. In Proceedings of the 2017 ACM International Conference on Interactive Surfaces and Spaces (Brighton, United Kingdom) (ISS '17). Association for Computing Machinery, New York, NY, USA, 280-286. https://doi.org/10.1145/3132272.3134140

[26] Chris Harrison, Hrvoje Benko, and Andrew D. Wilson. 2011. OmniTouch: Wearable Multitouch Interaction Everywhere. In Proceedings of the 24th Annual ACM Symposium on User Interface Software and Technology (Santa Barbara, California, USA) (UIST '11). Association for Computing Machinery, New York, NY, USA, 441-450. https://doi.org/10.1145/2047196.2047255

[27] Chris Harrison, Desney Tan, and Dan Morris. 2010. Skinput: Appropriating the Body as an Input Surface. In Proceedings of the SIGCHI Conference on Human Factors in Computing Systems (Atlanta, Georgia, USA) (CHI '10). Association for Computing Machinery, New York, NY, USA, 453-462. https://doi.org/10.1145/ 1753326.1753394

[28] Steven J. Henderson and Steven Feiner. 2008. Opportunistic Controls: Leveraging Natural Affordances as Tangible User Interfaces for Augmented Reality. In Proceedings of the 2008 ACM Symposium on Virtual Reality Software and Technology (Bordeaux, France) (VRST '08). Association for Computing Machinery, New York, NY, USA, 211-218. https://doi.org/10.1145/1450579.1450625

[29] Seongkook Heo, Michelle Annett, Benjamin Lafreniere, Tovi Grossman, and George Fitzmaurice. 2017. No Need to Stop What You'Re Doing: Exploring No-Handed Smartwatch Interaction. In Proceedings of the 43rd Graphics Interface Conference (Edmonton, Alberta, Canada) (GI '17). Canadian Human-Computer Communications Society, Waterloo, CAN, 107-114.

[30] Anuruddha Hettiarachchi and Daniel Wigdor. 2016. Annexing Reality: Enabling Opportunistic Use of Everyday Objects as Tangible Proxies in Augmented Reality. In Proceedings of the 2016 CHI Conference on Human Factors in Computing Systems (San Jose, California, USA) (CHI '16). Association for Computing Machinery, New York, NY, USA, 1957-1967. https://doi.org/10.1145/2858036.2858134

[31] Fang Hu, Peng He, Songlin Xu, Yin Li, and Cheng Zhang. 2020. FingerTrak: Continuous 3D Hand Pose Tracking by Deep Learning Hand Silhouettes Captured by Miniature Thermal Cameras on Wrist. Proc. ACM Interact. Mob. Wearable Ubiquitous Technol. 4, 2, Article 71 (June 2020), 24 pages. https://doi.org/10.1145/ 3397306

[32] Edwin Hutchins, James Hollan, and Donald Norman. 1985. Direct Manipulation Interfaces. Human-computer Interaction 1 (12 1985), 311-338. https://doi.org/10. 1207/s15327051hci0104_2 
[33] Yasha Iravantchi, Yang Zhang, Evi Bernitsas, Mayank Goel, and Chris Harrison 2019. Interferi: Gesture Sensing Using On-Body Acoustic Interferometry. In Proceedings of the 2019 CHI Conference on Human Factors in Computing Systems (Glasgow, Scotland Uk) (CHI '19). Association for Computing Machinery, New York, NY, USA, Article 276, 13 pages. https://doi.org/10.1145/3290605.3300506

[34] Md. Anamul Islam, Kenneth Sundaraj, R.Badlishah Ahmad, Nizam Ahamed, and Md. Asraf Ali. 2013. Mechanomyography Sensor Development, Related Signal Processing, and Applications: A Systematic Review. Sensors fournal, IEEE 13 (07 2013), 2499-2516. https://doi.org/10.1109/JSEN.2013.2255982

[35] Andrés Jaramillo-Yánez, Marco E. Benalcázar, and Elisa Mena-Maldonado. 2020 Real-Time Hand Gesture Recognition Using Surface Electromyography and $\mathrm{Ma}-$ chine Learning: A Systematic Literature Review. Sensors 20, 9 (2020). https: //doi.org/10.3390/s20092467

[36] Paul Kabbash, I. Scott MacKenzie, and William Buxton. 1993. Human Performance Using Computer Input Devices in the Preferred and Non-Preferred Hands. In Proceedings of the INTERACT '93 and CHI '93 Conference on Human Factors in Computing Systems (Amsterdam, The Netherlands) (CHI '93). Association for Computing Machinery, New York, NY, USA, 474-481. https://doi.org/10.1145/ 169059.169414

[37] David Kim, Otmar Hilliges, Shahram Izadi, Alex D. Butler, Jiawen Chen, Iason Oikonomidis, and Patrick Olivier. 2012. Digits: Freehand 3D Interactions Anywhere Using a Wrist-Worn Gloveless Sensor. In Proceedings of the 25th Annual ACM Symposium on User Interface Software and Technology (Cambridge, Massachusetts, USA) (UIST '12). Association for Computing Machinery, New York, NY, USA, 167-176. https://doi.org/10.1145/2380116.2380139

[38] Eddy Krueger, Eduardo MendonÃ Scheeren, Guilherme Nunes Nogueira-Neto, Vera LÃ da Silveira Nantes Button, and Percy Nohama. 2014. Advances and perspectives of mechanomyography. Revista Brasileira de Engenharia Biom ̃̃ 30 (12 2014), $384-401$. http://www.scielo.br/scielo.php?script=sci arttext\&pid= $\mathrm{S} 1517-31512014000400009 \& \mathrm{nrm}=$ iso

[39] Wakaba Kuno, Maki Sugimoto, and Yuta Sugiura. 2019. Method of estimating hand posture by measuring skin deformation of the back of the hand. Fournal of the Institute of Image Information and Television Engineers 73, 3 (2019), 595-601. https://doi.org/10.3169/itej.73.595

[40] Gierad Laput and Chris Harrison. 2019. Sensing fine-grained hand activity with smartwatches. Conference on Human Factors in Computing Systems - Proceedings. https://doi.org/10.1145/3290605.3300568

[41] Gierad Laput, Robert Xiao, and Chris Harrison. 2016. ViBand: High-Fidelity BioAcoustic Sensing Using Commodity Smartwatch Accelerometers. In Proceedings of the 29th Annual Symposium on User Interface Software and Technology (Tokyo, Japan) (UIST '16). Association for Computing Machinery, New York, NY, USA, 321-333. https://doi.org/10.1145/2984511.2984582

[42] Gierad Laput, Chouchang Yang, Robert Xiao, Alanson Sample, and Chris Harrison. 2015. EM-Sense: Touch Recognition of Uninstrumented, Electrical and Electromechanical Objects. In Proceedings of the 28th Annual ACM Symposium on User Interface Software \& Technology (Charlotte, NC, USA) (UIST '15). Association for Computing Machinery, New York, NY, USA, 157-166. https //doi.org/10.1145/2807442.2807481

[43] Darren Leigh, Clifton Forlines, Ricardo Jota, Steven Sanders, and Daniel Wig dor. 2014. High Rate, Low-Latency Multi-Touch Sensing with Simultaneous Orthogonal Multiplexing. In Proceedings of the 27th Annual ACM Symposium on User Interface Software and Technology (Honolulu, Hawaii, USA) (UIST '14). Association for Computing Machinery, New York, NY, USA, 355-364. https: //doi.org/10.1145/2642918.2647353

[44] X. Liang, H. Heidari, and R. Dahiya. 2017. Wearable Capacitive-Based WristWorn Gesture Sensing System. In 2017 New Generation of CAS (NGCAS). 181-184 https://doi.org/10.1109/NGCAS.2017.80

[45] Jhe-Wei Lin, Chiuan Wang, Yi Yao Huang, Kuan-Ting Chou, Hsuan-Yu Chen, Wei-Luan Tseng, and Mike Y. Chen. 2015. BackHand: Sensing Hand Gestures via Back of the Hand. In Proceedings of the 28th Annual ACM Symposium on User Interface Software \&; Technology (Charlotte, NC, USA) (UIST '15). Association for Computing Machinery, New York, NY, USA, 557-564. https://doi.org/10.1145/ 2807442.2807462

[46] Jia Liu, Fangxiaoyu Feng, Yuzuko C. Nakamura, and Nancy S. Pollard. 2014. A taxonomy of everyday grasps in action. 2014 IEEE-RAS International Conference on Humanoid Robots (2014), 573-580.

[47] M. K. Liu, Y. T. Lin, Z. W. Qiu, C. K. Kuo, and C. K. Wu. 2020. Hand Gesture Recognition by a MMG-Based Wearable Device. IEEE Sensors fournal 20, 24 (2020), 14703-14712. https://doi.org/10.1109/JSEN.2020.3011825

[48] Pedro Lopes, Patrik Jonell, and Patrick Baudisch. 2015. Affordance++: Allowing Objects to Communicate Dynamic Use. In Proceedings of the 33rd Annual ACM Conference on Human Factors in Computing Systems (Seoul, Republic of Korea) (CHI '15). Association for Computing Machinery, New York, NY, USA, 2515-2524. https://doi.org/10.1145/2702123.2702128

[49] Yiqin Lu, Bingjian Huang, Chun Yu, Guahong Liu, and Yuanchun Shi. 2020. Designing and Evaluating Hand-to-Hand Gestures with Dual Commodity WristWorn Devices. Proc. ACM Interact. Mob. Wearable Ubiquitous Technol. 4, 1, Article 20 (March 2020), 27 pages. https://doi.org/10.1145/3380984
[50] I. Scott Mackenzie. 2003. Motor Behaviour Models for Human-Computer Interaction (1 ed.). San Francisco, CA, USA

[51] Andualem Tadesse Maereg, Yang Lou, Emanuele Secco, and Raymond King. 2019. Hand Gesture Recognition Based on Near-Infrared Sensing Wristband. https://doi.org/10.5220/0008909401100117

[52] Jess McIntosh, Asier Marzo, and Mike Fraser. 2017. SensIR: Detecting Hand Gestures with a Wearable Bracelet Using Infrared Transmission and Reflection. In Proceedings of the 30th Annual ACM Symposium on User Interface Software and Technology (Québec City, QC, Canada) (UIST '17). Association for Computing Machinery, New York, NY, USA, 593-597. https://doi.org/10.1145/3126594.3126604

[53] Jess McIntosh, Charlie McNeill, Mike Fraser, Frederic Kerber, Markus Löchtefeld, and Antonio Krüger. 2016. EMPress: Practical Hand Gesture Classification with Wrist-Mounted EMG and Pressure Sensing. In Proceedings of the $2016 \mathrm{CHI}$ Conference on Human Factors in Computing Systems (San Jose, California, USA) (CHI '16). Association for Computing Machinery, New York, NY, USA, 2332-2342. https://doi.org/10.1145/2858036.2858093

[54] Manuel Meier, Paul Streli, Andreas Fender, and Christian Holz. 2021. TapID: Rapid Touch Interaction in Virtual Reality using Wearable Sensing. In 2021 IEEE Conference on Virtual Reality and 3D User Interfaces (VR) (Lisbon, Portugal) (IEEE $V R$ '21). IEEE.

[55] Edward F. Melcer, Michael T. Astolfi, Mason Remaley, Adam Berenzweig, and Tudor Giurgica-Tiron. 2018. CTRL-Labs: Hand Activity Estimation and RealTime Control from Neuromuscular Signals. In Extended Abstracts of the 2018 CHI Conference on Human Factors in Computing Systems (Montreal QC, Canada) (CHI EA '18). Association for Computing Machinery, New York, NY, USA, 1-4. https://doi.org/10.1145/3170427.3186520

[56] F. Pedregosa, G. Varoquaux, A. Gramfort, V. Michel, B. Thirion, O. Grisel, M. Blondel, P. Prettenhofer, R. Weiss, V. Dubourg, J. Vanderplas, A. Passos, D. Cournapeau, M. Brucher, M. Perrot, and E. Duchesnay. 2011. Scikit-learn: Machine Learning in Python. Fournal of Machine Learning Research 12 (2011), 2825-2830.

[57] Henning Pohl and Michael Rohs. 2014. Around-Device Devices: My Coffee Mug is a Volume Dial. In Proceedings of the 16th International Conference on HumanComputer Interaction with Mobile Devices \& Services (Toronto, ON, Canada) (MobileHCI '14). Association for Computing Machinery, New York, NY, USA, 81-90. https://doi.org/10.1145/2628363.2628401

[58] Francis Quek, David McNeill, Robert Bryll, Susan Duncan, Xin-Feng Ma, Cemil Kirbas, Karl E. McCullough, and Rashid Ansari. 2002. Multimodal Human Discourse: Gesture and Speech. ACM Trans. Comput.-Hum. Interact. 9, 3 (Sept. 2002), 171-193. https://doi.org/10.1145/568513.568514

[59] Hanae Rateau, Laurent Grisoni, and Bruno De Araujo. 2014. Mimetic interaction spaces : Controlling distant displays in pervasive environments. International Conference on Intelligent User Interfaces, Proceedings IUI, 89-94. https://doi.org/ $10.1145 / 2557500.2557545$

[60] Jun Rekimoto. 2001. GestureWrist and GesturePad: Unobtrusive Wearable Interaction Devices. In Proceedings of the 5th IEEE International Symposium on Wearable Computers (ISWC '01). IEEE Computer Society, USA, 21.

[61] Jaime Ruiz and Yang Li. 2011. DoubleFlip: A Motion Gesture Delimiter for Mobile Interaction. In Proceedings of the SIGCHI Conference on Human Factors in Computing Systems (Vancouver, BC, Canada) (CHI '11). Association for Computing Machinery, New York, NY, USA, 2717-2720. https://doi.org/10.1145/1978942. 1979341

[62] Valkyrie Savage, Sean Follmer, Jingyi Li, and Björn Hartmann. 2015. Makers' Marks: Physical Markup for Designing and Fabricating Functional Objects. In Proceedings of the 28th Annual ACM Symposium on User Interface Software \& Technology (Charlotte, NC, USA) (UIST '15). Association for Computing Machinery, New York, NY, USA, 103-108. https://doi.org/10.1145/2807442.2807508

[63] G. Schlesinger. 1919. Der mechanische Aufbau der künstlichen Glieder. Springer Berlin Heidelberg, Berlin, Heidelberg, 321-661. https://doi.org/10.1007/978-3662-33009-8_13

[64] Adwait Sharma, Joan Sol Roo, and Jürgen Steimle. 2019. Grasping Microgestures: Eliciting Single-Hand Microgestures for Handheld Objects. In Proceedings of the 2019 CHI Conference on Human Factors in Computing Systems (Glasgow, Scotland Uk) (CHI '19). Association for Computing Machinery, New York, NY, USA, Article 402, 13 pages. https://doi.org/10.1145/3290605.3300632

[65] Jennifer G. Sheridan and Gerd Kortuem. 2006. Affordance-Based Design of Physical Interfaces for Ubiquitous Environments. In Proceedings of the Third International Conference on Ubiquitous Computing Systems (Seoul, Korea) (UCS'06). Springer-Verlag, Berlin, Heidelberg, 183-199. https://doi.org/10.1007/11890348 15

[66] Mohamed Soliman, Franziska Mueller, Lena Hegemann, Joan Sol Roo, Christian Theobalt, and Jürgen Steimle. 2018. FingerInput: Capturing Expressive Single-Hand Thumb-to-Finger Microgestures. In Proceedings of the 2018 ACM International Conference on Interactive Surfaces and Spaces (Tokyo, Japan) (ISS '18). Association for Computing Machinery, New York, NY, USA, 177-187. https://doi.org/10.1145/3279778.3279799

[67] Charles W. Suggs. 1969. The Effect of Load on Muscle Output. Human Factors 11, 3 (1969), 273-279. https://doi.org/10.1177/001872086901100309 arXiv:https://doi.org/10.1177/001872086901100309 
[68] Yuta Sugiura, Fumihiko Nakamura, Wataru Kawai, Takashi Kikuchi, and Maki Sugimoto. 2017. Behind the palm: Hand gesture recognition through measuring skin deformation on back of hand by using optical sensors. In 2017 56th Annual Conference of the Society of Instrument and Control Engineers of Japan, SICE 2017, Vol. 2017-November. Institute of Electrical and Electronics Engineers Inc., 1082-1087. https://doi.org/10.23919/SICE.2017.8105457

[69] Mihai T Tarata. 2003. Biomedical Engineering Online 2 (02 2003), 3. https: //dx.doi.org/10.1186/1475-925X-2-3

[70] Hoang Truong, Shuo Zhang, Ufuk Muncuk, Phuc Nguyen, Nam Bui, Anh Nguyen, Qin Lv, Kaushik Chowdhury, Thang Dinh, and Tam Vu. 2018. CapBand: BatteryFree Successive Capacitance Sensing Wristband for Hand Gesture Recognition. In Proceedings of the 16th ACM Conference on Embedded Networked Sensor Systems (Shenzhen, China) (SenSys '18). Association for Computing Machinery, New York, NY, USA, 54-67. https://doi.org/10.1145/3274783.3274854

[71] Velko Vechev, Alexandru Dancu, Simon T. Perrault, Quentin Roy, Morten Fjeld, and Shengdong Zhao. 2018. Movespace: On-Body Athletic Interaction for Running and Cycling. In Proceedings of the 2018 International Conference on Advanced Visual Interfaces (Castiglione della Pescaia, Grosseto, Italy) (AVI '18). Association for Computing Machinery, New York, NY, USA, Article 28, 9 pages. https://doi.org/10.1145/3206505.3206527

[72] Daniel Vogel and Ravin Balakrishnan. 2005. Distant Freehand Pointing and Clicking on Very Large, High Resolution Displays. In Proceedings of the 18th Annual ACM Symposium on User Interface Software and Technology (Seattle, WA USA) (UIST '05). Association for Computing Machinery, New York, NY, USA 33-42. https://doi.org/10.1145/1095034.1095041

[73] Julie Wagner, Mathieu Nancel, Sean G. Gustafson, Stephane Huot, and Wendy E Mackay. 2013. Body-Centric Design Space for Multi-Surface Interaction. In Proceedings of the SIGCHI Conference on Human Factors in Computing Systems (Paris, France) (CHI '13). Association for Computing Machinery, New York, NY, USA, 1299-1308. https://doi.org/10.1145/2470654.2466170

[74] James A. Walsh, Stewart von Itzstein, and Bruce H. Thomas. 2014. Ephemeral Interaction Using Everyday Objects. In Proceedings of the Fifteenth Australasian User Interface Conference - Volume 150 (Auckland, New Zealand) (AUIC '14). Australian Computer Society, Inc., AUS, 29-37.

[75] Mark Weiser. 1999. The Computer for the 21st Century. SIGMOBILE Mob. Comput Commun. Rev. 3, 3 (July 1999), 3-11. https://doi.org/10.1145/329124.329126

[76] Hongyi Wen, Julian Ramos Rojas, and Anind K. Dey. 2016. Serendipity: Finger Gesture Recognition Using an Off-the-Shelf Smartwatch. In Proceedings of the 2016 CHI Conference on Human Factors in Computing Systems (San Jose, California, USA) (CHI '16). Association for Computing Machinery, New York, NY, USA 3847-3851. https://doi.org/10.1145/2858036.2858466
[77] Andrew D. Wilson and Hrvoje Benko. 2010. Combining Multiple Depth Cameras and Projectors for Interactions on, above and between Surfaces. In Proceedings of the 23nd Annual ACM Symposium on User Interface Software and Technology (New York, New York, USA) (UIST '10). Association for Computing Machinery, New York, NY, USA, 273-282. https://doi.org/10.1145/1866029.1866073

[78] Erwin Wu, Ye Yuan, Hui-Shyong Yeo, Aaron Quigley, Hideki Koike, and Kris M. Kitani. 2020. Back-Hand-Pose: 3D Hand Pose Estimation for a Wrist-Worn Camera via Dorsum Deformation Network. In Proceedings of the 33rd Annual ACM Symposium on User Interface Software and Technology (Virtual Event, USA) (UIST '20). Association for Computing Machinery, New York, NY, USA, 1147-1160. https://doi.org/10.1145/3379337.3415897

[79] Xing-Dong Yang, Tovi Grossman, Daniel Wigdor, and George Fitzmaurice. 2012. Magic Finger: Always-Available Input through Finger Instrumentation. In Proceedings of the 25th Annual ACM Symposium on User Interface Software and Technology (Cambridge, Massachusetts, USA) (UIST '12). Association for Computing Machinery, New York, NY, USA, 147-156. https://doi.org/10.1145/2380116.2380137

[80] Tom Yeh, Tsung-Hsiang Chang, and Robert C. Miller. 2009. Sikuli: Using GUI Screenshots for Search and Automation. In Proceedings of the 22nd Annual ACM Symposium on User Interface Software and Technology (Victoria, BC, Canada) (UIST '09). Association for Computing Machinery, New York, NY, USA, 183-192. https://doi.org/10.1145/1622176.1622213

[81] Hui-Shyong Yeo, Erwin Wu, Juyoung Lee, Aaron Quigley, and Hideki Koike. 2019. Opisthenar: Hand Poses and Finger Tapping Recognition by Observing Back of Hand Using Embedded Wrist Camera. In Proceedings of the 32nd Annual ACM Symposium on User Interface Software and Technology (New Orleans, LA, USA) (UIST '19). Association for Computing Machinery, New York, NY, USA, 963-971. https://doi.org/10.1145/3332165.3347867

[82] Takatoshi Yoshida, Xiaoyan Shen, Tal Achituv, and Hiroshi Ishii. 2018. 3D Touch Point Detection on Load Sensitive Surface Based on Continuous Fluctuation of a User Hand. In SIGGRAPH Asia 2018 Posters (Tokyo, Japan) (SA '18). Association for Computing Machinery, New York, NY, USA, Article 39, 2 pages. https: //doi.org/10.1145/3283289.3283339

[83] Yang Zhang and Chris Harrison. 2015. Tomo: Wearable, Low-Cost Electrical Impedance Tomography for Hand Gesture Recognition. In Proceedings of the 28th Annual ACM Symposium on User Interface Software \& Technology (Charlotte, NC, USA) (UIST '15). Association for Computing Machinery, New York, NY, USA, 167-173. https://doi.org/10.1145/2807442.2807480

[84] Qian Zhou, Sarah Sykes, Sidney Fels, and Kenrick Kin. 2020. Gripmarks: Using Hand Grips to Transform In-Hand Objects into Mixed Reality Input. In Proceedings of the 2020 CHI Conference on Human Factors in Computing Systems (Honolulu, HI, USA) (CHI '20). Association for Computing Machinery, New York, NY, USA, 1-11. https://doi.org/10.1145/3313831.3376313 